\title{
AmpC hyperproduction in a Cedecea davisae implant-associated bone infection during treatment: a case report and therapeutic implications
}

Julia Notter ${ }^{1 *}+\left(\mathbb{0}\right.$, Salome N. Seiffert ${ }^{2 \dagger}$, Maria Zimmermann-Kogadeeva ${ }^{3}$, Anja Bösch ${ }^{4}$, Robert Wenger ${ }^{5}$, Carol Strahm ${ }^{1}$, Manuel Frischknecht ${ }^{1}$, David M. Livermore ${ }^{6}$ and Baharak Babouee Flury ${ }^{1,4}$

\begin{abstract}
Background: Data on antimicrobial resistance mechanisms are scanty for Cedecea spp., with very variable antibiotic resistance patterns documented. Here we report the first in vivo resistance evolution of a C. davisae clinical isolate in a patient with a complex hand trauma and provide insight in the resistance mechanism, leading to therapeutic implications for this pathogen.

Case presentation: Cedecea davisae was isolated from a patient with hand trauma during a first surgical debridement. Six days after primary surgical treatment and under antimicrobial treatment with amoxicillin-clavulanic acid and later cefepime, follow up cultures yielded $C$. davisae which demonstrated a resistance development. The susceptible parental isolate and its resistant derivative were characterized by whole genome sequencing, $\mathrm{ampC}$, ompC and ompF by RT- PCR. The resistant derivative demonstrated an A224G SNP in ampD, the transcriptional regulator of ampC, leading to a His75Arg change in the corresponding AmpD protein. AmpC transcription of the resistant derivative was 362-times higher than the susceptible isolate. Transcription levels of ompF and ompC were 8.5-fold and 1.3-fold lower, respectively, in the resistant derivative. Downregulation of $\mathrm{OmpF}$ putatively resulted from a mutation in the presumed promoter region upstream of the dusB-Fis operon, a proposed regulator for ompF.
\end{abstract}

Conclusions: This case demonstrates the in vivo resistance development of $C$. davisae within 7 days similar to that of the members of the Enterobacter cloacae complex. Our findings add valuable information for future therapeutic management of these opportunistic pathogens as they warrant the same empirical treatment as AmpC producers.

Keywords: Cedecea davisae, AmpC, Hyperproducing, Cefepime, Resistance evolution, Case report, C. davisae implantassociated bone infection

*Correspondence: Julia.notter@kssg.ch

† Julia Notter and Salome N. Seiffert contributed equally to this work

${ }^{1}$ Division of Infectious Diseases and Hospital Epidemiology, Kantonsspital

St. Gallen, St. Gallen, Switzerland

Full list of author information is available at the end of the article

\section{Background}

Cedecea spp. are Gram-negative bacilli belonging to the Enterobacterales [1]. They can act as opportunistic pathogens, principally in immunocompromised hosts, with $C$. davisae, C. lapagei and C. neteri all documented as having clinical significance [2]. Although infections are infrequent and sporadic, reports are increasing [2]. Recent papers indicate 13 case reports of $C$. davisae infections 
to date, starting from 1977. Infection sites include blood, sputum, gall bladder, skin wounds and abscesses [2, 3]. More than three quarters of the patients were $\geq 50$ years of age, and most were severely immunocompromised, with multiple comorbid diseases [2]. Very variable antibiograms have been documented for the genus: resistances to amoxicillin, amoxicillin-clavulanate and cephalosporins are frequent, though not universal [2]. Data on resistance mechanisms are scarce. Acquired New Delhi metallo- $\beta$-lactamase-1 (NDM-1) has been detected in C. lapagei and $C$. davisae $[2,3]$. Perhaps of greater general significance, a novel AmpC $\beta$-lactamase was characterized from a $C$. davisae clinical isolate in 2014 [3]; this resembled the chromosomal AmpC $\beta$-lactamases of Enterobacter spp. and was non-transferable.

Here we report in vivo evolution of $\beta$-lactam resistance in a $C$. davisae implant-associated bone infection, characterized by whole genome sequencing. Expression of $a m p C, o m p C$ and $o m p F$ was assayed by Reverse Transcriptase PCR (RT-PCR). Our findings add valuable information for future therapeutic management of these opportunistic pathogens.

\section{Case presentation}

A 33-year-old man with a history of curatively-treated seminoma presented to our emergency room with skin and soft tissue necrosis on his right hand, along with increasing pain, 1 day after being discharged from an external hospital (Fig. 1). Two weeks previously he had suffered a complex right-hand trauma while cleaning an industrial flour mixer. The external hospital had immediately performed an initial surgery, involving osteosynthesis and tendon repair. Due to a type III open fracture he had received an empirical treatment with amoxicillinclavulanic acid (6 g/day i.v.) until discharge.

Following presentation at our hospital amoxicillin-clavulanic acid (6 g/day i.v.) was restarted; it was assumed that the tissue necrosis was caused by poor blood circulation (Fig. 1). Since the patient's symptoms did not improve, debridement, necrosectomy and transmetacarpal amputation of the index finger and partial removal of osteosynthesis material were performed 6 days after presentation (Day 6, Fig. 2). Amoxicillin-clavulanic acid was continued for 4 days post-surgery, until samples, taken on the day of surgery, revealed the growth of C. davisae resistant to this agent (Table 1). Anaerobic cultures were also performed and yielded no growth. Antimicrobial treatment was then switched to cefepime (6 g/d i.v.), based on a concern that $C$. davisae might have a potential to overexpress an AmpC enzyme. Two days after switch to cefepime, a new "second-look" debridement surgery was performed (Day 12, Fig. 2). Cultures at this time again yielded $C$. davisae but with additional resistance to

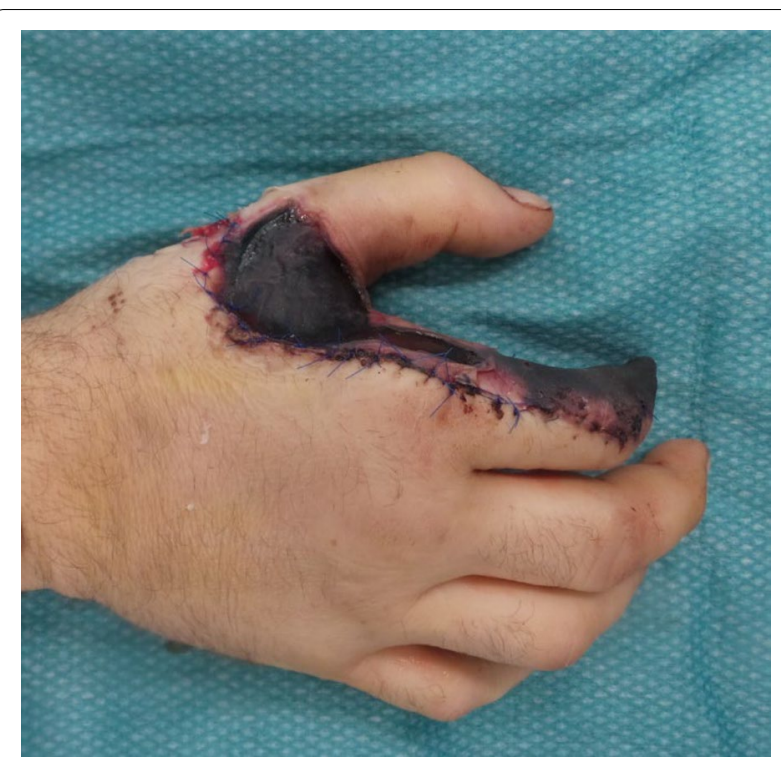

Fig. 1 Clinical presentation at the emergency room at our hospital

ceftriaxone, ceftazidime, piperacillin-tazobactam and a raised 'on the breakpoint' MIC for ertapenem $(0.5 \mathrm{mg} / \mathrm{L}$, Table 1). While precise MIC data for cefepime were pending, antibiotic therapy was switched to meropenem ( $3 \mathrm{~g} /$ day i.v.) and a reconstruction using a radial forearm flap was undertaken to close the defect and cover the exposed bone and remaining ostheosynthesis material. Subsequent testing showed that the cefepime MIC for the strain had also increased, though only from 0.047 to $1 \mathrm{mg} / \mathrm{L}$.

The patient thereafter showed a satisfactory course and was 2 weeks later released into outpatient care with oral trimethoprim-sulfamethoxazole $(3 \mathrm{~g} / \mathrm{d})$ for further 6 months. There were no signs of a recurrent infection 4 weeks after stopping the antibiotic therapy. The plan is to remove the remaining osteosynthesis material in a further surgery and to treat the underlying osteomyelitis with ciprofloxacin (Fig. 2).

\section{Microbiological testing}

All samples from the patient were processed in November 2020 according to the accredited routine procedures of the Centre for Laboratory Medicine in St. Gallen, Switzerland. Identification was with MALDI-ToF mass spectrometry (Bruker Daltonics, Bremen, Germany) using the BDAL 9.0 database; routine susceptibility testing was performed with the NMIC-417 panel on the BD Phoenix ${ }^{\text {TM }}$ M50 (Becton Dickinson, Franklin Lakes, NJ, USA). Further broth microdilution testing using Sensititre GNX2F plates (Trek Diagnostic Systems, UK) with Mueller-Hinton broth (BBL, Becton Dickinson) was performed at the 


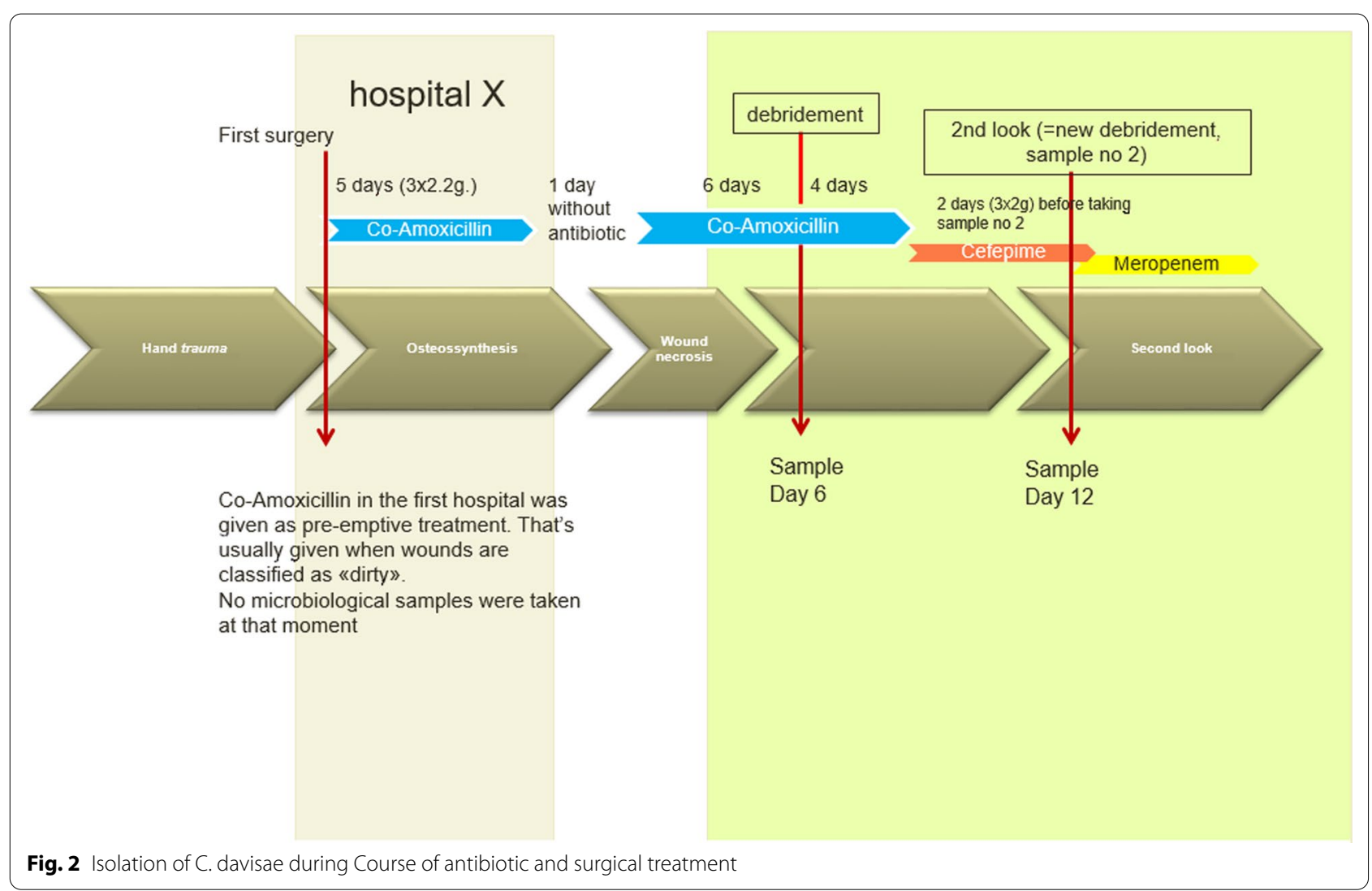

Table 1 Phenotypic susceptibility patterns of the two C. davisae isolates

\begin{tabular}{lll}
\hline Antibiotic & $\begin{array}{l}\text { Parental isolate } \\
\text { (Day 6:18.11.20) }\end{array}$ & $\begin{array}{l}\text { Resistant } \\
\text { isolate (Day 12: } \\
\mathbf{2 4 . 1 1 . 2 0 )}\end{array}$ \\
\hline Amoxicillin-clavulanic acid & $32(\mathrm{R})$ & $>64(\mathrm{R})$ \\
Piperacillin-tazobactam & $\leq 6(\mathrm{~S})$ & $32(\mathrm{R})$ \\
Cefotaxime & $\leq 0.5(\mathrm{~S})$ & $>16(\mathrm{R})$ \\
Ceftazidime & $\leq 0.5(\mathrm{~S})$ & $>16(\mathrm{R})$ \\
Cefepime & $\leq 1 ; 0.047^{\mathrm{a}}(\mathrm{S})$ & $\leq 1 ; 1^{\mathrm{a}}(\mathrm{S})$ \\
Aztreonam & $\leq 1(\mathrm{~S})$ & $>8(\mathrm{R})$ \\
Imipenem & $\leq 0.5(\mathrm{~S})$ & $\leq 0.5(\mathrm{~S})$ \\
Meropenem & $\leq 0.5(\mathrm{~S})$ & $\leq 0.5(\mathrm{~S})$ \\
Ertapenem & $\leq 0.19(\mathrm{~S})$ & $0.5(\mathrm{~S})$ \\
Ciprofloxacin & $\leq 0.19(\mathrm{~S})$ & $\leq 0.19(\mathrm{~S})$ \\
Co-Trimoxazole & $\leq 0.25(\mathrm{~S})$ & $\leq 0.25(\mathrm{~S})$ \\
Gentamicin & $\leq 0.5(\mathrm{~S})$ & $\leq 0.5(\mathrm{~S})$ \\
Tobramycin & $\leq 0.5(\mathrm{~S})$ & $\leq 0.5(\mathrm{~S})$ \\
\hline S, susepible &
\end{tabular}

$S$, susceptible, R, resistant according to EUCAST guidelines (version 10) a By Etest

Medical Research Centre. In the case of cefepime, precise 'on-scale' MICs were determined by Etest (bioMérieux, Marcy l'Etoile, France). Antimicrobial susceptibility data were interpreted according to EUCAST guidelines (version 10.0, $2020[4])$.

\section{Whole genome sequencing and mutation analysis}

The Day 6 isolate and its resistant Day 12 counterpart were characterized by whole genome sequencing (WGS). DNA extraction was performed using the QIAsymphony DSP DNA Mini Kit (QIAGEN GmbH, Hilden, Germany); sequencing with an Illumina MiSeq instrument and the Nextera XT library preparation kit (Illumina Inc., USA); all were used according to the manufacturers' procedures. Assembly was performed using the Ridom Seqsphere + Software with standard settings (Ridom: Munster, Germany). Both genomes had over $40 \times$ coverage (NCBI accession numbers: SAMN18652104 and SAMN18652105). Annotation was performed using the Prokka software (version 1.14.6) [5]; For SNP detection, the susceptible parental isolate was used as a reference, and calling was conducted using Snippy (version 4.6.0) [6].

\section{Evaluation of transcription levels}

Reverse transcriptase (RT)-PCR was used to measure mRNA levels for $b l a_{A m p C}$, ompF and ompC (Fig. 3), using the primers listed in Table 2. Mid-logarithmic phase 


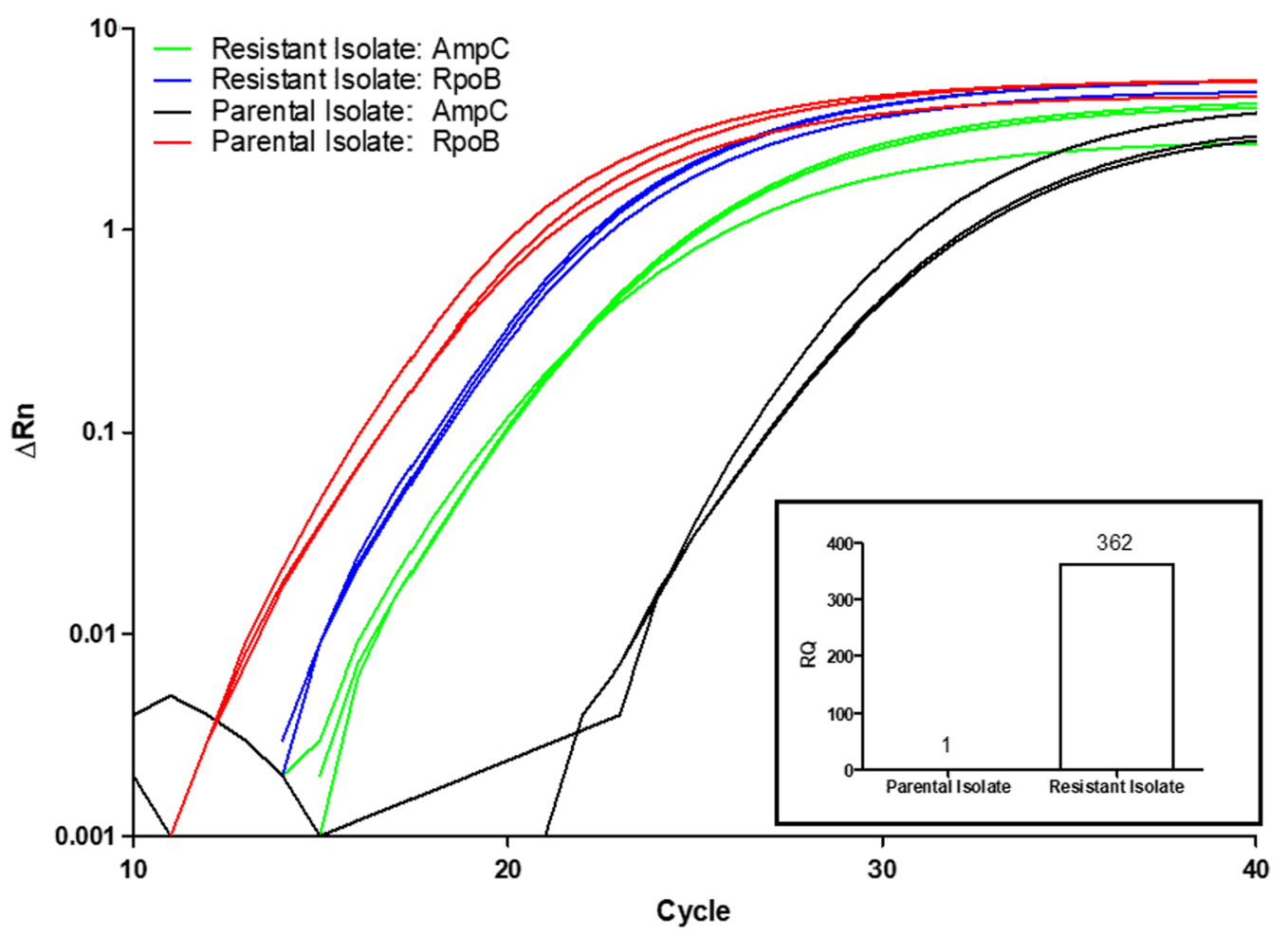

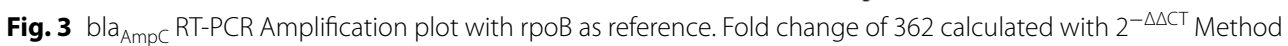

Table 2 Primers used for RT-PCR expression analysis

\begin{tabular}{lll}
\hline Name & Sequence & Reference \\
\hline RT Ceda RpoB_F2 & 5'TGA CAA GCT CGA CAA ACT GC 3' & This study \\
RT Ceda RpoB_R2 & 5'CGC CCT GAG TGA TTT TAC GG 3' & This study \\
RT Ceda AmpC_F1 & 5'AGT GCT GGA ACC ATT GAA GC 3' & This study \\
RT Ceda AmpC_R1 & 5'TTC GAT GCT GGA CTT AAC GC 3' & This study \\
RT Ceda OmpC_F2 & 5'TGT TAC CTG CGG CAT CAT TG 3' & This study \\
RT Ceda OmpC_R2 & 5'GCT ATG AGT CCC AGG GCTTT 3' & This study \\
RT Ceda OmpF_F2 & 5'CCG TAC CAA TGC CCA ACA AA 3' & This study \\
RT Ceda OmpF_R2 & 5'AGT GCT GCC AGG TAG ATG TT 3' & This study \\
\hline
\end{tabular}

cultures $(0.5 \mathrm{ml})$ of the Day 6 and 12 C. davisae isolates were treated with the RNAprotect reagent (Qiagen). RNA was then extracted with an RNeasy Mini Kit (Qiagen) and the eluate treated with DNase I (Qiagen), used according to the manufacturer's instruction. RT-PCR was subsequently performed using the Power SYBR ${ }^{\circledR}$ Green RNA-to-CT 1-Step Kit (Thermo Fisher Scientific, Vilnius, Lithuania) and a QuantStudio 5 Real-Time PCR System (Applied Biosystems by Thermo Fisher Scientific) at an annealing temperature of $60{ }^{\circ} \mathrm{C}$. Transcript measurements were carried out in triplicate and measurements were repeated twice. Quantification of relative target gene expression was by the $2^{-\Delta \Delta C T}$ method, using $r p o B$ as a reference, as described previously [7]. The original Day 6 C. davisae isolate was used as the calibrator (Table 2).

\section{Microbiological results}

Susceptibility data for the Day 6 and Day 12 isolates are summarized in Table 1. Both isolates were resistant to amoxicillin/clavulanate and both susceptible to cefepime, imipenem, meropenem and various non- $\beta$-lactams. They differed in that the Day 6 isolate was susceptible to ceftriaxone, ceftazidime and piperacillin/tazobactam whereas the Day 12 isolate was resistant to these agents and had reduced susceptibility to ertapenem. The cefepime MIC for the Day 12 isolate, by Etest, was 21-fold higher than for the Day 6 isolate $(1 \mathrm{mg} / \mathrm{L}$ vs. $0.047 \mathrm{mg} / \mathrm{L}$, Table 1$)$ but remained in EUCAST's susceptible range [4]. Except for SNPs, detailed below, the two isolates were identical by WGS, confirming that they represented the same strain.

The Day 12 derivative had an A224G SNP in $a m p D$, the transcriptional regulator of $a m p C$, leading to a His75Arg change in the corresponding AmpD protein. Correlating with this, AmpC transcription in the resistant derivative was 362-times higher than the Day 6 isolate (Fig. 3). There were no mutations within ompF and ompC; 


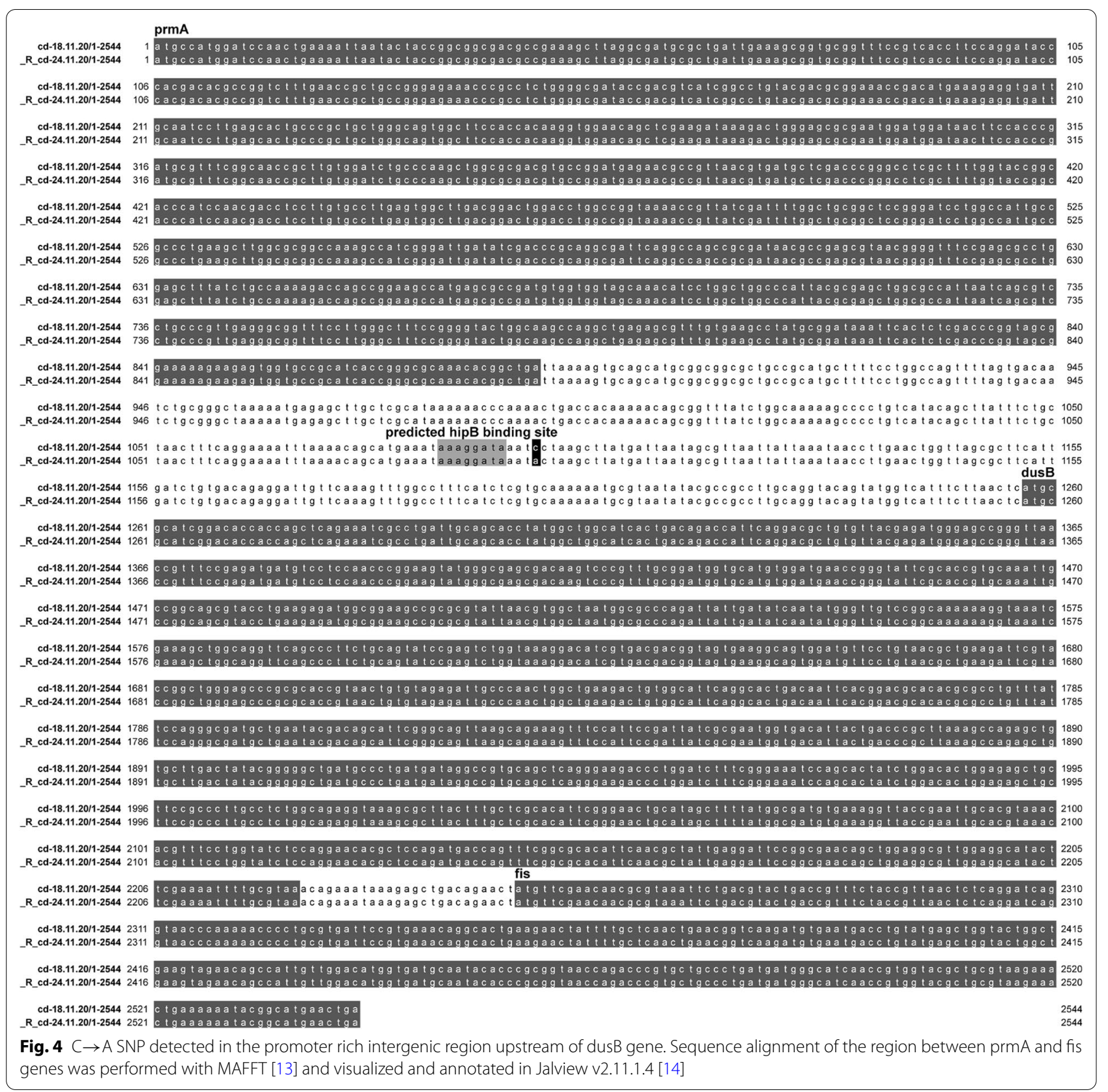

however, transcription levels of these outer membrane proteins were 8.5 -fold and 1.3-fold lower, respectively, in the resistant derivative.

Six further SNPs distinguished the parent and the resistant organisms, potentially explaining these latter differences. Three of these SNPs were in intergenic regions (Table 3 ) and one $(\mathrm{C} \rightarrow \mathrm{A})$ was 162 nucleotides upstream of $d u s B$, which belongs to the $d u s B$-fis operon, where Fis is a transcriptional regulator reported to affect expression of ompF [8]. Notably, this SNP was located in a potentially promoter-rich intergenic region, four nucleotides downstream of a predicted helix-turn-helix transcription factor $h i p B$ binding site, as found using the Softberry [9] (Fig. 4).

\section{Discussion and conclusion}

This case demonstrates that resistance to $\beta$-lactams can develop in $C$. davisae via mutation of $a m p D$, leading to hyperproduction of the AmpC $\beta$-lactamase, as also frequently occurs e.g. in members of the Enterobacter cloacae complex [10]. Although AmpC inducibility was not investigated, an $a m p R$ homologue was found by 
Table 3 SNPs between the parent (Day 6) and the resistant (Day 12) isolates

\begin{tabular}{|c|c|c|c|c|c|c|}
\hline Contig & Position & Day 6 & Day 12 & Effect & Gene & Product \\
\hline 5 & 184001 & A & G & & & \\
\hline 11 & 91073 & $\mathrm{~T}$ & C & $\begin{array}{l}\text { Missense_variant A224G } \\
\text { p.His75Arg }\end{array}$ & $a m p D$ & $\begin{array}{l}\text { 1;6-anhydro-N-acetylmuramyl-L-alanine } \\
\text { amidase AmpD }\end{array}$ \\
\hline 13 & 36350 & A & G & $\begin{array}{l}\text { Missense_variant } \\
\text { T614C } \\
\text { p.Val205Ala }\end{array}$ & yicl & Putative inner membrane transporter YicL \\
\hline 35 & 57393 & A & G & $\begin{array}{l}\text { Missense_variant T1645 >C } \\
\text { p.Cys549Arg }\end{array}$ & hemR & Hemin receptor \\
\hline 41 & 87395 & CCCC & TCCA & $\begin{array}{l}\text { Missense_variant 602_605delCCC- } \\
\text { CinsTCCA p.ThrPro201IleHis }\end{array}$ & mdoC & Glucan biosynthesis protein $C$ \\
\hline 41 & 576365 & G & A & & & \\
\hline 54 & 159944 & C & A & & & Intergenic region upstream of $d u s B$ gene \\
\hline
\end{tabular}

p. corresponding amino acid change

NCBI Accession Numbers: parent isolate (day 6): SAMN18652104; resistant isolate (day 12): SAMN18652105

sequencing upstream the $a m p C$, and an $a m p R-a m p C$ operon, predicting inducibility and the increased risk of selecting hyperproducers [11], has been described previously in the related species, C. neteri [12]. We suggest that the additional rise in ertapenem MIC seen here reflected downregulation of OmpF, putatively as a result of mutation in the presumed promoter region upstream of the dusB-Fis operon, a proposed regulator for OmpF.

Resistance to $\beta$-lactams, including carbapenems, has been associated previously with a combination of AmpC activity and loss of both porins OmpC and OmpF in $C$. davisae [3] but the in-vivo evolution of resistance associated with these mechanisms has not been recorded in the literature. It is perhaps surprising that this evolution occurred with sequential use of amoxicillin-clavulanate acid, which lacked activity against even the initial isolate, and cefepime, which retained activity even against the second isolate, albeit with a raised MIC. Our findings should inform future therapeutic management of infections due to these uncommon opportunistic pathogens, underscoring that they warrant the same caution as other species where AmpC derepression is a hazard.

\section{Abbreviations}

MALDI-ToF: Matrix-assisted laser desorption/ionization time-of-flight-mass spectrometry; MIC: Minimal inhibitory concentration; NDM-1: New Delhi metallo- $\beta$-lactamase-1; RT-PCR: Reverse Transcriptase PCR; SNP: Single nucleotide polymorphism; WGS: Whole genome sequencing.

\section{Acknowledgements}

Not applicable.

\section{Authors' contributions}

$\mathrm{JN}, \mathrm{CS}, \mathrm{MF}$ and RW were involved in patient management and collection of patient data. JN and AB carried out laboratory experiments, SNS and MZK performed the molecular analyses, JN, SNS and BBF wrote the manuscript with input from all authors, DML and BBF conceived the experiments and were involved in patient management. All authors read and approved the final manuscript.

\section{Funding}

This study was funded by a research grant of the Medical Research Centre of Kantonsspital St. Gallen, Switzerland. The funding body had no role in the design of the study and collection, analysis, and interpretation of data and in writing the manuscript.

\section{Availability of data and materials}

Whole genome sequences of the isolates are available on NCBI Accession Numbers: SAMN18652104 and SAMN18652105.

\section{Declarations}

Ethics approval and consent to participate

The patient signed an informed consent allowing the publication of his case description including clinical pictures.

\section{Consent for publication}

The patient signed an informed consent allowing the publication of his case description including clinical pictures.

\section{Competing interests}

The authors declare that they have no competing interests.

\section{Author details}

${ }^{1}$ Division of Infectious Diseases and Hospital Epidemiology, Kantonsspital St. Gallen, St. Gallen, Switzerland. ${ }^{2}$ Division of Human Microbiology, Centre for Laboratory Medicine, St. Gallen, Switzerland. ${ }^{3}$ Genome Biology Unit, European Molecular Biology Laboratory (EMBL), Heidelberg, Germany. ${ }^{4}$ Medical Research Centre, Kantonsspital St. Gallen, St. Gallen, Switzerland. ${ }^{5}$ Division of Hand, Plastic and Reconstructive Surgery, Kantonsspital St. Gallen, St. Gallen, Switzerland. ${ }^{6}$ Norwich Medical School, University of East Anglia, Norwich, UK.

Received: 5 August 2021 Accepted: 21 December 2021

Published online: 06 January 2022

\section{References}

1. Nakhoul F. A rare bacteremia caused by Cedecea davisae in patient with chronic renal disease. Am J Case Rep. 2013;14:216-8.

2. Thompson DK, Sharkady SM. Expanding spectrum of opportunistic Cedecea infections: current clinical status and multidrug resistance. Int J Infect Dis. 2020;100:461-9.

3. Ammenouche N, Dupont H, Mammeri H. Characterization of a novel AmpC $\beta$-lactamase produced by a carbapenem-resistant Cedecea davisae clinical isolate. Antimicrob Agents Chemother. 2014;58(11):6942-5. 
4. The European Committee on Antimicrobial Susceptibility Testing. Breakpoint tables for interpretation of MICs and zone diameters, version 10.0, 2020. http://www.eucast.org/clinical_breakpoints/.

5. Seeman T. Prokka: rapid prokaryotic genome annotation. Bioinformatics. 2014;30(14):2068-9.

6. Seemann T. Fast bacterial variant calling from NGS reads. Verfügbar unter: https://github.com/tseemann/snippy.

7. Livak KJ, Schmittgen TD. Analysis of relative gene expression data using real-time quantitative $P C R$ and the $2-\triangle \triangle C T$ method. Methods. 2001;25(4):402-8.

8. Crozat E, Hindré T, Kühn L, Garin J, Lenski RE, Schneider D. Altered regulation of the OmpF porin by Fis in Escherichia coli during an evolution experiment and between B and K-12 strains. J Bacteriol. 2011;193(2):429-40

9. Solovyev V, Salamov A. Automatic annotation of microbial genomes and metagenomic sequences. In: Metagenomics and its applications in agriculture, biomedicine and environmental studies. Nova Science Publishers; 2011. Verfügbar unter: http://www.softberry.com/berry.phtml.

10. Babouee Flury B, Ellington MJ, Hopkins KL, Turton JF, Doumith M, Loy R, et al. Association of novel nonsynonymous single nucleotide polymorphisms in ampD with cephalosporin resistance and phylogenetic variations in ampC, ampR, ompF, and ompC in Enterobacter cloacae isolates that are highly resistant to carbapenems. Antimicrob Agents Chemother. 2016:60(4):2383-90

11. Jacoby GA. AmpC $\beta$-lactamases. CMR. 2009;22(1):161-82.

12. Ginn PS, Tart SB, Sharkady SM, Thompson DK. Urinary catheter colonization by multidrug-resistant Cedecea neteri in patient with Benign prostatic hyperplasia. Case Rep Infect Dis. 2018;2018:1-5.

13. Katoh K, Rozewicki J, Yamada KD. MAFFT online service: multiple sequence alignment, interactive sequence choice and visualization. Brief Bioinform. 2019:20(4):1160-6.

14. Waterhouse AM, Procter JB, Martin DMA, Clamp M, Barton GJ. Jalview Version 2-a multiple sequence alignment editor and analysis workbench. Bioinformatics. 2009;25(9):1189-91.

\section{Publisher's Note}

Springer Nature remains neutral with regard to jurisdictional claims in published maps and institutional affiliations.

Ready to submit your research? Choose BMC and benefit from:

- fast, convenient online submission

- thorough peer review by experienced researchers in your field

- rapid publication on acceptance

- support for research data, including large and complex data types

- gold Open Access which fosters wider collaboration and increased citations

- maximum visibility for your research: over $100 \mathrm{M}$ website views per year

At BMC, research is always in progress.

Learn more biomedcentral.com/submissions 